\title{
Overcoming the UCB HSCs -Derived NK Cells Dysfunctionality through Harnessing RAS/MAPK, IGF-1R and TGF- $\beta$ Signaling Pathways
}

\section{Alireza Shokouhifar}

Royan Institute for Stem Cell Biology and Technology https://orcid.org/0000-0002-9693-4101

Gholamreza Anani Sarab

Birjand university of medical sciences

\section{Mahboubeh Yazdanifar}

Stanford university school of medicine

Mohammad Fereidouni

Birjand University of Medical Sciences

\section{Masoumeh Nouri}

Royan Institute for Stem Cell Biology and Technology

Marzieh Ebrahimi ( $\nabla$ mebrahimi@royaninstitute.org )

Cell Science Research Center, Royan Institute for Stem Cell Biology and Technology, ACECR, Tehran, Iran https://orcid.org/0000-0003-1140-0379

Primary research

Keywords: Umbilical Cord blood, Natural killer cells, Differentiation, Cytotoxicity, Cancer Immunotherapy

Posted Date: November 3rd, 2020

DOl: https://doi.org/10.21203/rs.3.rs-98506/v1

License: (c) (1) This work is licensed under a Creative Commons Attribution 4.0 International License.

Read Full License 


\section{Abstract}

\section{Background}

Natural killer (NK) cells differentiated from umbilical cord blood (UCB) hematopoietic stem cells (HSCs) may be more suitable for cell-based immunotherapy compared to NK cells from adult donors. This is due to opportunity to choose alloreactive donors and potentially more robust in vivo expansion. However, the cytotoxicity of UCB-HSC derived NK cells against cancer cells might be suboptimal. To overcome this obstacle, we attempted to generate NK cells with potent antitumor activity by targeting RAS/MAPK, IGF$1 \mathrm{R}$ and TGF- $\beta$ signaling pathways.

\section{Methods}

The CD34+ cells isolated from human UCB mononuclear cells through MACS with purity of ( $\geq 90 \%)$ were used to be differentiated into NK cells. After 21 days of induction with SFTG36, IS721 and IL-15/Hsp70 media, NK cells phenotype was studied and their cytotoxicity against K562 human erythroleukemia cell and SKOV3 ovarian carcinoma cells was analyzed.

\section{Results}

The induced NK cells treated with SFTG36/I721 and SFTG36/IS721 growth factor cocktail expressed a phenotype with CD56+16+CD3- and NKG2D+ with mean fluorescence intensity (MFI) of 92.7\% \pm 1.45 $168.00 \pm 19.20$ and $93.23 \% \pm 0.75-168.66 \pm 20.00$ respectively. These NK cells once activated by IL-15, demonstrated a higher cytotoxicity against K562 ( $\geq 90 \%)(P \leq 0.001)$ and SKOV3 tumor cells $(\geq 65 \%)(P$ $\leq 0.001)$ compared to IL-15/Hsp70 activated NK cells.

\section{Conclusion}

The differentiation of ex vivo-expanded CD34+ cells through manipulation of RAS/MAPK, IGF-1R and TGF- $\beta$ signaling pathways is an efficient approach for generating functional NK cells that can be used for cancer immunotherapy.

\section{Highlights}

- NK cells are innate immune cells with broad anti-tumor activity. Due to not being restricted to a specific human leukocyte antigen (HLA) as T cells and not causing graft versus host disease (GvHD), NK cells are ideal candidate for developing off-the-shelf adoptive cell immunotherapy for a wide range of cancers.

- A major obstacle in NK cells clinical application is their poor ex vivo expansion and proliferation.

- NK cells can be drawn from different sources such as peripheral blood, NK cell lines and umbilical cord blood (UCB). Generating NK cells via differentiating UCB hematopoietic stem cells (HSCs) is a more suitable approach for cell-based immunotherapy compared to deriving NK cells from adult 
donors. This is due to the opportunity to choose alloreactive donors and potentially more robust in vivo expansion from a pluripotent progenitor.

- We could successfully generate functional NK cells from UCB-HSC. To improve their cytotoxicity against cancer cells, we targeted RAS/MAPK, IGF-1R and TGF- $\beta$ signaling pathways during NK cells differentiation and expansion phases.

- In this study we demonstrated an optimal protocol for the differentiation of ex vivo-expanded CD34+ cells through manipulation of RAS/MAPK, IGF-1R and TGF- $\beta$ signaling pathways as an efficient approach for generating functional NK cells for cancer immunotherapy.

\section{Introduction}

Immune cell-based therapy aims at harnessing the patient's own immune system to fight cancer and to obtain long-term responses in patients (Lim, Jung, Hwang, \& Shin, 2015; Souza-Fonseca-Guimaraes, Cursons, \& Huntington, 2019). Among various components of the immune system, natural killer (NK) cells are innate immune system lymphocytes with ability to kill the tumor and virus-infected cells without prior sensitization to antigens (Farag \& Caligiuri, 2006; Gardiner, 2019). Although many efforts have been expended to date to apply NK cells derived from peripheral blood to adoptive therapy of solid and hematopoietic cancers (Klingemann, 2015; Lim et al., 2015); this approach has encountered several pitfalls such as insufficient cell number, limited source accessibility, lower response to stimulants, decreased expansion potential and increased dysfunctionality. To address these limitations, other sources for NK cells such as bone marrow, embryonic cells, induced pluripotent and umbilical cord blood stem cells (UCB-HSC) have been tested (Briard, Brouty-Boye, Azzarone, \& Jasmin, 2002; Mehta, Shpall, \& Rezvani, 2015; Mu et al., 2019; Pinho, Punzel, Sousa, \& Barros, 2011; Spanholtz et al., 2011).

UCB-HSCs have significant advantages including easy expansion and differentiation into other immune cells, containing numerous NK cell progenitors, fast engraftment and immune reconstitution, less stringent requirements for HLA matching and lower risk of graft versus host disease (GvHD); hence, they can be used to generate NK cells (Borrego, Ulbrecht, Weiss, Coligan, \& Brooks, 1998; Lim et al., 2015; Mehta et al., 2015). Various methods have been developed to generate NK cells with highly purity (>90\%) from UCB-HSCs (Briard et al., 2002; Mehta et al., 2015; Mu et al., 2019; Pinho et al., 2011; Spanholtz et al., 2011). Although several clinical trials have been performed; it is still difficult to obtain functional NK cells by ex vivo cultivation of UCB-HSCs (Mehta et al., 2015; Mu et al., 2019). The dysfunctional NK cells phenotypes are often determined as downregulation of specific surface activating receptors on NK cells in tumors and chronic infections (Judge, Murphy, \& Canter, 2020). NKG2D is often downregulated on NK cells in patients with different types of malignancies. Following the downregulation of NKG2D activating receptor and its signaling adaptors, DAP10/12, other activating receptors such as CD16 and NCRs (NKp30, NKp44 and NKp46) also show a decreased expression in different types of tumors and chronic infection which represents compelling evidence for a synergic effect of the activating receptor's signaling pathways in NK cells (Bi \& Tian, 2017; Judge et al., 2020; Vely \& Vivier, 2005). 
Moreover, surface receptors inducing inhibitory signals in NK cells, such as NKG2A are overexpressed in malignancies and chronic infections (Chester, Fritsch, \& Kohrt, 2015; Judge et al., 2020). NKG2A is one of the most important inhibitory receptors which plays a role in the dysfunctionality of NK cells in the patients with the mentioned diseases through inducing IL-10 production. Other inhibitory receptors having a prominent role in NK cells inactivation are Tim3 and PD-1 (Chan, Smyth, \& Martinet, 2014; Judge et al., 2020; Vivier, Nunes, \& Vely, 2004; Watzl \& Long, 2010).

RAS/MAPK, PI3K/AKT, JAK/STAT and TGF- $\beta$ are among the most prominent signaling pathways involved in switching between NK activation and inhibition (Chan et al., 2014; Judge et al., 2020; Vely \& Vivier, 2005). Therefore targeting these pathways may be an effective strategy for producing active and nondysfunctional NK cells.

The aim of the present study is to generate functional NK cells and in order to achieve this goal, we have manipulated the critical signaling pathways for activation and inhibition of NK cells starting from the early differentiation phase from UCB-CD34+ (Fig. 5). Another goal of this study is achieving the desirable cell quantity of about 600 fold expansion. In the present study, we demonstrated that NK cells differentiated from UCB-CD34 + cells have a significant reduction in dysfunctionality characteristics, high expansion potential and robust anti-tumor cytotoxicity against K562 and SKOV3 tumor cells.

\section{Materials And Methods Cell lines}

K562 and SKOV3 cell lines (Royan Institute Cell Bank, Tehran, Iran) were cultured in RPMI 1640 (Gibco, USA) containing $50 \mathrm{U} / \mathrm{mL}$ penicillin, $50 \mathrm{mg} / \mathrm{mL}$ streptomycin and $10 \%$ fetal calf serum.

\section{Isolation and expansion of CD34+ cells}

UCB units were obtained from the Cord Blood Bank, Royan Stem Cell Technology Co. with the following ethical codes: IR.BUMS.REC.1396.321 and IR.ACECR.ROYAN.REC.1397.171. MNCs were harvested from UCB using Ficoll-Hypaque density gradient centrifugation and CD34+ progenitor cells were then isolated from freshly isolated UCB MNCs through magnetic bead selection (Miltenyi Biotech, USA). The CD34+ cells were seeded at $7 \times 10^{5}$ cells per well in 6-well plates (TPP, Switzerland) in Advanced RPMI 1640 medium (Gibco, USA) with penicillin/streptomycin $(100 \mathrm{U} / \mathrm{mL})$ and cytokines cocktail (SCF, TPO, FLt3-L, IL-3, IL-6, and GM-CSF) (all from R\&D Systems, USA) at the expansion phase of cultures (Table 1). Half of the media was replaced with fresh media every 2 to 3 days for 7 days.

\section{Differentiation of CD34+ cells to NK cells and their activation}


The expanded CD34+ cells were cultured in the NK cell differentiation medium supplemented with SCF, TPO, rhIGF-1 (Royan Biotech CO, Iran), rhIL7, rhIL21 and SIS3 small molecule (smad3 inhibitor)

(Calbiochem, USA) at the density of $7 \times 10^{5}$ cells per well in 6-well plates and half of the induction medium was changed every 2-3 days. The differentiation medium that was used from day 7 to day 17 .

An activation medium containing SCF, TPO, rhIGF-1, rhIL7, rhIL21, SIS3 small molecule (smad3 inhibitor), rhIL15 and rhHsp70 (according to our previous study ((Sharifzad et al., 2020)) was used. The details of culture media used in each phase are listed in Table 1.

Fold expansion was calculated by dividing the absolute output number of the expanded cells after each phase of culture by the respective number on the day 0 and the immunophenotyping was determined by flow cytometry.

\section{Colony forming unit assay (CFU assay)}

The CFU assay was performed using commercially available methylcellulose medium (MethoCult H4435 Enriched; Stem cell Technologies, Vancouver, BC, Canada). This medium supports the clonal progeny of a single-cell to differentiate and grow in distinct colonies including BFU-E (Burst-forming unit - erythroid), CFU-E (Colony-forming unit - erythroid), CFU-GEMM (Colony-forming unit - granulocyte, erythroid, macrophage, megakaryocyte) and CFU-GM (Colony-forming unit - granulocyte, macrophage). $1 \times 10^{3}$ CD34+ cells were added to $2 \mathrm{ml}$ MethoCult $\mathrm{H} 4435$ medium, vortexed vigorously until the cells were well suspended and $1 \mathrm{ml}$ of the cell suspension was subsequently plated in a 12-well plate at a final density of $5 \times 10^{2}$ cells/well. Cells were finally incubated at $37^{\circ} \mathrm{C} 5 \% \mathrm{CO} 2$ for $12-14$ days.

\section{Wright-Giemsa staining}

Morphological characteristics of the expanded CD34+ cells were detected by Wright-Giemsa staining on the day 14. Briefly smears of cells were stained with Wright-Giemsa solution for 25 minutes, rinsed with distilled water and air dried. Cells morphology was studied by light microscopy.

\section{Flow cytometry analysis}

The prepared single cell solutions were labelled with a combination of antibodies including CD45-FITC, CD34-PE, CD3-FITC and CD56/CD16-PE (all from BD Biosciences, USA). In the expansion phase, cells were characterized as CD34+ cells and in the differentiation phase, NK cells were characterized as CD3CD56+/CD16+ cells.

\section{Quantitative real time reverse transcription PCR}


Perforin, IFN-y, Granzyme A and B mRNA levels were evaluated by quantitative real-time RT-PCR (SYBR Green assay) using a Rotor-Gene 6000 (Corbett Life Sciences, Sydney, Australia) Sequence Detector. RNAs were isolated using Gene All Hybrid-R kit (Biotech, Korea). Spectrophotometric methods were used to assess the quality and quantity of the RNAs. CDNA was synthesized from $1 \mu \mathrm{g}$ of total RNA with RT-forPCR kit (Takara Bio, Inc., Otsu, Japan) according to the manufacturer's instructions. The sequence of primers used for measuring gene expression is listed in supplementary table 1. $\beta 2$-microglubolin RNA levels was used as an internal control for all experiments. Amplification was performed for 1 cycle of a sequential incubation at $50^{\circ} \mathrm{C}$ for $2 \mathrm{~min}, 95^{\circ} \mathrm{C}$ for $10 \mathrm{~min}$, and subsequent 40 cycles of a consecutive incubations at $94^{\circ} \mathrm{C}$ for $1 \mathrm{~min}, 60^{\circ} \mathrm{C}$ for $1 \mathrm{~min}$ and $72^{\circ} \mathrm{C}$ for $1.5 \mathrm{~min}$. The individual gene expression value was calculated after normalization to $\beta 2$-microglubolin gene.

Quantitation of the mRNAs was determined by the comparative cycle threshold (CT) method. The relative change in mRNA concentration for each test sample was then determined from the difference between the calibrator CT and the CT of each test sample. Each sample was run in triplicate. The relative expression of the target genes was determined using $2^{-\Delta \Delta C t}$ equation.

\section{Cytotoxicity assay}

K562 and SKOV3 cells inactivated with Mitomycin C $(10 \mathrm{ug} / \mathrm{mL})$ were labeled with Calcein AM by incubating for $45 \mathrm{~min}$ at $37^{\circ} \mathrm{C}$ in $5 \% \mathrm{CO} 2$. Cells were then washed with PBS, re-suspended in RPMI with $10 \%$ FBS, and plated in 6-well plates at $3 \otimes 10^{5}$ cells/well in triplicate. Effector cells were co-cultured with K562 cells at effector:target (E:T) ratios of 5:1 and 3:1 followed by incubation for 24, 48 and 72 hours at $37^{\circ} \mathrm{C}$ in $5 \% \mathrm{CO} 2$, and at 3:1 E:T ratio for SKOV3 cells and incubated for 48 hours at $37^{\circ} \mathrm{C}$ in $5 \% \mathrm{CO} 2$. Cytotoxicity level was evaluated by Calcein/propidium iodide (PI) staining and flowcytometry.

\section{Statistical analysis}

The statistical analysis were performed using Graph pad Prism Ver.8 and the data were presented as the means \pm SEM. Statistical differences were determined using paired Student's t-tests or two-tailed Student's t-tests when comparing two groups and one-way or two-way ANOVA analysis when comparing more than two groups. Differences were considered statistically significant at ${ }^{*} p<0.05,{ }^{\star \star} p<0.01,{ }^{\star \star \star} p<0.001$ and $\star \star \star \star p<00.0001$.

\section{Results}

\section{Choosing the proper cytokine cocktail for the expansion of UCB hematopoietic CD34 + progenitor}

In the first step, we sought to select the best cocktail of cytokines for CD34 + cells expansion with strong effect on NK cell differentiation. Different combination of cytokines were selected based on literature and 
divided into two groups: SFTG36 medium containing SCF/FLt3-L/TPO/GM-CSF/IL-3/IL-6 and SFT medium containing SCF/FLt3-L/TPO. SFTG36 medium was more effective in generating CFU-BFU-E, CFUGM and CFU-GEMM after 14 days of cultivation in MethoCult H4435 medium $(p<0.0001)$ (more colonies and larger) (Fig. 1-A, B). The obtained results was also confirmed morphologically through Wright-Giemsa staining (more lymphoid progenitors) (Fig. 1-A). Purified CD34 + cells (with purity of $92 \% \pm 2.30$ )

(Supplementary Fig. 1) were expanded by 53.72 fold (range: 33.7 to 91.8 ) in SFTG36 and by 8.00 fold (range: 3.60 to 11.93) in SFT media during 7 days of cultivation (Fig. 1-C). Cell viability was evaluated using Trypan Blue and Calcein/PI staining and results did not show any significant difference between SFT and SFTG36 groups. Both groups demonstrated a viability higher than 95\% (Fig. 1-D).

Cells expanded in SFTG36 medium revealed higher percentage of CD $45+$ cells $(79.55 \% \pm 18.2)$ and CD45+/CD34 + cells $(42.33 \% \pm 19.00)$ compared to SFT group (Fig. 1-E); therefore, cells cultivated in SFTG36 medium were selected for NK differentiation.

\section{The growth factor cocktail augments the efficacy of NK cell differentiation}

As mentioned earlier, different signaling pathways affect NK cell differentiation and the efficacy of differentiation procedure. In the present study, to promote effective NK differentiation, RAS/MAPK pathway was activated using IGF-1 (Ni et al., 2013), JAK/STAT signaling pathway was induced using IL21 (Seo et al., 2017; Skak, Frederiksen, \& Lundsgaard, 2008) and TGF- $\beta$ signaling pathway was blocked using SIS3 small molecule (Jinnin, Ihn, \& Tamaki, 2006; Tang et al., 2017; Wang et al., 2018). Moreover, IL7 was used as the NK cells differentiation and maturation factor (Michaud et al., 2010; Wu, Tian, \& Wei, 2017). Therefore, the expanded HSCs cells were cultivated in 1721 medium containing IL-7/IL-21 + IGF-1 and IS721 medium containing IL-7/IL-21 + IGF-1 + SIS3.

Our results indicated a low percentage of CD3 + and CD3+/CD $56+C D 16+$ in all tested groups $(<1 \%$ and $<2 \%$, respectively) following 17 days of cultivation (Supplementary Fig. 2). Therefore, it can be concluded that the expanded CD34 + cells have not been differentiated into T cells. On the other hand, cells treated with both conditions represented the highest percentage of CD56 + 16 + CD3- cells (92.7\% \pm 1.45 in 1721 and $93.23 \% \pm 0.75$ in IS721 group) (Fig. 2-A, B). Expression of NKG2D activating marker also demonstrated a significant increase in the MFI (168.00 \pm 19.20 in 1721 and $168.66 \pm 20.00$ in IS721 group) (Fig. 2-A, C and Supplementary Fig. 2).

Differentiated cells also continued their expansion in this step and the cells treated with $1721(8.093 \pm$ $1.205)$ and IS721 demonstrated a more significant expansion (11.893 \pm 1.712$)$ compared to the other group (Fig. 2-D). 


\section{Cell lysis functional assay and gene expression analysis of induced NK cells}

To induce cytotoxic activity of the differentiated NK cells, they were cultivated in the presence of IL-15 and IL-15/Hsp70 for additional four days. Therefore, the differentiated NK cells in IS721 group were selected for activation. Immunophenotyping analysis revealed a higher increase for NKp44 expression in the group treated with IL-15 $(92.2 \% \pm 0.30)$, while no significant difference was observed in the expression of NKG2D, NKG2A and NKp30 markers (Fig. 3- A- Left). However, MFI of NKG2D (61.12 $\pm 17.62, P<$ $0.0001)$ and NKp30 (48.17 $\pm 0.83, P<0.0001)$ were higher in IL-15/Hsp70 treated group compared to IL15 alone (Fig. 3- A- Right).

Quantitative gene expression analysis between IL-15 and IL-15 + Hsp70 groups demonstrated a higher fold change in all genes expression (decrease in gene expression level) including perforin, IFN- $y$, granzyme A and B in IL-15 + Hsp70 group compared to IL-15 group as 0.317 fold $(P<0.0001), 0.280$ fold $(P<0.01), 0.323$ fold $(P<0.0001)$ and 0.160 fold $(P<0.001)$, respectively. (Fig. $3-\mathrm{B})$.

The cytotoxic function of NK cells against K562 erythroleukemia cells was surveyed as the gold standard of NK cells activity assay. To perform this assay, NK cells activated by the above mentioned activating combinations, were co-cultured with K562 in 5:1 and 3:1 E:T ratios and the cell lysis potential of NK cells was analyzed in 24, 48 and 72 hours following the co-culture (Fig. 4- A and B). NK cells treated with IL-15 in 5:1 ratio and 24, 48 and 72 hours after co-culture with K562 tumor cells demonstrated the highest cell lysis (>89\%, $P<0.001)$ compare to $3: 1$ ratio $(>85 \%)$, but NK cells treated with IL-15 with the $5: 1$ ratio showed the highest cell lysis capability in 24 hours following the co-culture $(>94 \%, P<0.0001$ ) (Fig. 4-Aleft and B). The comparison of cytotoxicity of the cells treated with IL-15 + Hsp70 in 24 hours after coculture in $5: 1$ ratio $(>87 \%, P<0.01)$ demonstrated the highest difference among groups (Fig. 4-A- right and B).

According to this data, NK cells treated with IL-15 were more successful in tumor cells lysis compared to the ones treated with IL-15/Hsp70, and 5:1 ratio had the highest significance among groups (Fig. 4-B). However, the group treated with IL-15 in 5:1 ratio showed the highest cytotoxicity among groups after 24 hours of co-culture with K562 tumor cells $(>94 \%, P<0.0001)$ (Fig. 4-A and B).

The cytotoxic capacity of the NK cells treated with IL-15 and IL-15 + Hsp70 against SKOV3 tumor cells was also assessed as a solid tumor model. According to our data, 3:1 ratio and 48 hours led to the highest level of cell lysis between the two groups; however, the cells treated with IL-15 showed significantly higher levels of SKOV3 tumor cells lysis $(>62 \%, P<0.0001)$ compared to the group treated with IL-15 + Hsp70 (> 17.27\%, $P<0.001)$ (Fig. 4-C). Finally, it was observed that the differentiated NK cells can retain their cytolytic potential and play their antitumor role for up to 72 hours post co-culture.

\section{Discussion}


In the present study, we demonstrated a highly efficient cell culture protocol for ex vivo generation of functional NK cell products from UCB-HSCs through targeting RAS/MAPK, IGF-1R and TGF-B pathways which can generate potentially functional and non-exhausted NK cells (Fig. 5). The cells obtained in this study have significant expansion potential of more than 600 folds (53 folds in expansion and 12 folds in differentiation phase) (Fig. 1-C and 2-D) due to the use of lower doses of the following molecules $(2.5 \mathrm{ng} / \mathrm{ml} \mathrm{SCF}, 2.5 \mathrm{ng} / \mathrm{ml} \mathrm{TPO}, 5 \mathrm{ng} / \mathrm{ml}$ rhlGF-1, $10 \mathrm{ng} / \mathrm{ml} \mathrm{rhlL7,} 10 \mathrm{ng} / \mathrm{ml}$ rhlL21, $25 \mathrm{ng} / \mathrm{ml}$ SIS3 inhibitor, $50 \mathrm{ng} / \mathrm{ml} \mathrm{rhlL} 15$ and $20 \mu \mathrm{g} / \mathrm{ml}$ rhHsp70) compared to other studies (SCF $100 \mathrm{ng} / \mathrm{ml}$ and TPO $100 \mathrm{ng} / \mathrm{ml}$ ) (Chotinantakul, Prasajak, \& Leeanansaksiri, 2013; Denning-Kendall, Singha, Bradley, \& Hows, 2003; Herrera et al., 2001; Mourcin et al., 2005; Y. Zhang et al., 2019). Two aims have been considered for the present study: 1- Increasing and maintaining the proliferation capacity of NK cells and 2- Increasing the cell lysis potential with the lowest level of dysfunctionality. To achieve these goals, RAS/MAPK, PI3K/AKT, JAK/STAT and TGF-B pathways were manipulated with low doses of SFTG36/IS721/IL-15 (Fig. 5). In the present study, the differentiated NK cells displayed high expression of CD56 $(>95.00 \%)$ and several activating receptors such as NKG2D (>94.00\%), NKp30 (>86.00\%) and NKp44 (>86.00\%); while the expression of NKG2A $(<36.00 \%)$ inhibitory receptor was not elevated (Fig. 2- B-C and 3-A).

To date, various protocols have been employed for ex vivo expansion and differentiation of NK cells from HSC sources; however, there are still several technical hindrances despite using different combinations of cytokines with or without feeder cell lines, and the use of animal and human sera could lead to many issues including production of inefficient NK cell products (Dezell et al., 2012; Domogala et al., 2017; Hosseini, Ghasemzadeh, Kamalizad, \& Schwarer, 2017; Kao et al., 2007; Mu et al., 2019; Spanholtz et al., 2011; Spanholtz et al., 2010).

It has been shown that the expanded CD34 + cells could be differentiated into a NK cell product with an average purity of $40-60 \%$ after 5-7 weeks of culture (Kao et al., 2007; Mu et al., 2019; Spanholtz et al., 2011). Many studies have reached a calculated mean expansion rate of 300 -fold during the NK cell generation phase (Spanholtz et al., 2011; Spanholtz et al., 2010).

Several studies have reported a multiple cytokines-based culture method for up to 6 weeks to generate therapeutic NK cell products with high cytotoxicity function (10:1, 85\%) from CB-derived CD34 + HSCs (Pinho et al., 2011; Spanholtz et al., 2011; Spanholtz et al., 2010). In the present study, a 600 fold expansion of NK cells with purity of $>90 \%$ was demonstrated after 3 weeks of culture and the NK cells differentiated from UCB-CD34 + HSCs were assayed in 3:1 and 5:1 ratios so as to measure the cytotoxicity potential. These cells differentiated with a growth factor cocktail, demonstrated cell lysis of > $90 \%$ against K562 tumor cells and $>60 \%$ against SKOV3 tumor cells (Fig. 4 ).

Dysfunctionality is one of the fundamental problems encountered during differentiation and activation of NK cells. In most studies, mediums containing IL-2 are used for NK cells differentiation and activation (Alvarez et al., 2019; Bi \& Tian, 2017; Judge et al., 2020; Seo et al., 2017). Dysfunctionality can be induced using exogenous molecules regulating the NK cells functions, such as IL-2, IL-15 and IL-21. They do so by increasing the expression of co-inhibitory receptors (PD-1), inhibitory receptors (NKG2A) and upregulation 
of activating receptors like NKG2D. All these changes can impair the balance of activating/inhibitory functions of NK cells and consequently result in exhaustion (Gasteiger et al., 2013; Huenecke et al., 2010; Miller et al., 1997; Seo et al., 2017; Sharifzad et al., 2020; Skak et al., 2008). Other factors that can suppress NK cells function during differentiation and activation are suppressive cytokines such as TGF- $\beta$ which induces the downregulation of activating receptors (Bi \& Tian, 2017; Huenecke et al., 2010; Judge et al., 2020; Seo et al., 2017; C. Zhang et al., 2019). The results of this study indicated that the differentiated NK cells have low level of NKG2A inhibitory receptors which can be the reason why the cells function equally against K562 cells (Bi \& Tian, 2017; Judge et al., 2020; C. Zhang et al., 2019).

In addition, Hsp70 in previous studies have been shown to increase the induction of NK cells. The declined cytotoxic function of Hsp70-treated NK cells against K562 and SKOV3 cells in the present study (Fig. 4), although contrary, can be explained by the dual function of Hsp70 which can also promote the cancer cells viability (Gyrd-Hansen, Nylandsted, \& Jaattela, 2004; Multhoff, 2009; Qiao, Liu, \& Li, 2008; Sharifzad et al., 2020; Specht et al., 2015; Zorzi \& Bonvini, 2011). Furthermore, considering the qRT-PCR results, it can be claimed that treating with Hsp70 led to a reduction in the NK cell lysis capacity compared to the other group.

A previous study has reported the positive effect of IGF-1 on human NK cell cytotoxicity and assessed the ability of endogenous miR-483-3p to regulate IGF-1 expression in UCB HSCs -derived NK cells using antimiR-483-3p to upregulate IGF-1 mRNA and protein levels. However, the exact regulatory mechanism of IGF-1 in enhancing NK cell function remains unclear. It has been shown that IGF-1 promotes cytotoxic function of UCB HSCs -derived NK cells (Ni et al., 2013). But in this study, rhIGF-1 protein was used as an exogenous treat in $50 \mathrm{ng} / \mathrm{ml}$ for 14 days to induce insulin receptor-dependent pathways (RAS/MAPK and $\mathrm{PI} 3 \mathrm{~K} / \mathrm{AKT}$ ), and the results showed that this growth factor was effective in NK cells proliferation, maturation and activation.

In conclusion, the obtained results in the present study show that the NK cells derived via our method can induce significant lytic function against K562 (>90\%) and SKOV3 $(>60 \%)$ targets in the lower ratio of effector cells compared with other studies (5:1), suggesting ex vivo generated UCB HSCs -derived NK cells could be suitable candidate for designing adoptive NK cell-based therapy for cancer (Fig. 4).

This study is the first to use the combination of rhIGF-1 protein and TGF-B blockade to generate cytotoxic NK cells, and further studies are required on the other tumor cells as well as the in vivo models. It is also essential to optimize the activation pathways and compare the cell lysis activities between CB-derived NK cells and the peripheral blood-derived NK cells.

\section{Abbreviations}

CML: chronic myeloid leukemia;

UCB: Umbilical Cord blood; 
E:T: Effector-to-target;

HSCs: Hematopoietic stem cells;

IL: Interleukin;

MNCs: Mononuclear cells;

NK: Natural killer;

\section{Declarations}

\section{Acknowledgements}

The authors would like to acknowledge the cancer research center of cancer institute of Iran and Royan Stem Cell Technology Co. for funding this project.

\section{Funding}

This work was supported by cancer research center of cancer institute of Iran (Shams cancer charity, Grant No: 37312-202-01-97) and Royan Stem Cell Technology Co. (Grant No. RSCT96030201).

\section{Availability of data and materials}

The datasets used and analyzed during the current study are available from the corresponding author on reasonable request.

\section{Ethics approval and consent to participate}

All CB samples were obtained with prior consent and ethical committee approval from Cord Blood Bank of Royan Stem Cell Technology Co, and Royan Institute.

\section{Author Contributions}

Alireza Shokouhifar: conception and design, performed experiments, data analysis and interpretation, Collection and/or assembly of data, manuscript writing.

Mahboubeh Yazdanifar: experiments planning, interpretation.

Mohammad Fereidouni: experiments planning, interpretation. 
Masoumeh Nouri: experiments planning, interpretation.

Gholamreza Anani Sarab: conception and design, interpretation, financial support, provision of study material, discussed the results and commented on the manuscript, final approval of manuscript, Administrative support and supervision.

Marzieh Ebrahimi: conception and design, interpretation, financial support, provision of study material, discussed the results and commented on the manuscript, final approval of manuscript, Administrative support and supervision.

\section{References}

1. Alvarez, M., Simonetta, F., Baker, J., Pierini, A., Wenokur, A. S., Morrison, A. R., . . Negrin, R. S. (2019). Regulation of murine NK cell exhaustion through the activation of the DNA damage repair pathway. JCl Insight, 5. doi:10.1172/jci.insight.127729

2. Bi, J., \& Tian, Z. (2017). NK Cell Exhaustion. Front Immunol, 8, 760. doi:10.3389/fimmu.2017.00760

3. Borrego, F., Ulbrecht, M., Weiss, E. H., Coligan, J. E., \& Brooks, A. G. (1998). Recognition of human histocompatibility leukocyte antigen (HLA)-E complexed with HLA class I signal sequence-derived peptides by CD94/NKG2 confers protection from natural killer cell-mediated lysis. J Exp Med, 187(5), 813-818. doi:10.1084/jem.187.5.813

4. Briard, D., Brouty-Boye, D., Azzarone, B., \& Jasmin, C. (2002). Fibroblasts from human spleen regulate NK cell differentiation from blood CD34(+) progenitors via cell surface IL-15. J Immunol, 168(9), 4326-4332. doi:10.4049/jimmunol.168.9.4326

5. Chan, C. J., Smyth, M. J., \& Martinet, L. (2014). Molecular mechanisms of natural killer cell activation in response to cellular stress. Cell Death Differ, 21(1), 5-14. doi:10.1038/cdd.2013.26

6. Chester, C., Fritsch, K., \& Kohrt, H. E. (2015). Natural Killer Cell Immunomodulation: Targeting Activating, Inhibitory, and Co-stimulatory Receptor Signaling for Cancer Immunotherapy. Front Immunol, 6, 601. doi:10.3389/fimmu.2015.00601

7. Chotinantakul, K., Prasajak, P., \& Leeanansaksiri, W. (2013). Wnt1 Accelerates an Ex Vivo Expansion of Human Cord Blood CD34(+)CD38(-) Cells. Stem Cells Int, 2013, 909812. doi:10.1155/2013/909812

8. Denning-Kendall, P., Singha, S., Bradley, B., \& Hows, J. (2003). Cytokine expansion culture of cord blood CD34+ cells induces marked and sustained changes in adhesion receptor and CXCR4 expressions. Stem Cells, 21(1), 61-70. doi:10.1634/stemcells.21-1-61

9. Dezell, S. A., Ahn, Y. O., Spanholtz, J., Wang, H., Weeres, M., Jackson, S., . . Verneris, M. R. (2012). Natural killer cell differentiation from hematopoietic stem cells: a comparative analysis of heparinand stromal cell-supported methods. Biol Blood Marrow Transplant, 18(4), 536-545. doi:10.1016/j.bbmt.2011.11.023 
10. Domogala, A., Blundell, M., Thrasher, A., Lowdell, M. W., Madrigal, J. A., \& Saudemont, A. (2017). Natural killer cells differentiated in vitro from cord blood CD34(+) cells are more advantageous for use as an immunotherapy than peripheral blood and cord blood natural killer cells. Cytotherapy, 19(6), 710-720. doi:10.1016/j.jcyt.2017.03.068

11. Farag, S. S., \& Caligiuri, M. A. (2006). Human natural killer cell development and biology. Blood Rev, 20(3), 123-137. doi:10.1016/j.blre.2005.10.001

12. Gardiner, C. M. (2019). NK cell metabolism. J Leukoc Biol, 105(6), 1235-1242. doi:10.1002/JLB.MR0718-260R

13. Gasteiger, G., Hemmers, S., Firth, M. A., Le Floc'h, A., Huse, M., Sun, J. C., \& Rudensky, A. Y. (2013). IL2-dependent tuning of NK cell sensitivity for target cells is controlled by regulatory T cells. J Exp Med, 210(6), 1167-1178. doi:10.1084/jem.20122462

14. Gyrd-Hansen, M., Nylandsted, J., \& Jaattela, M. (2004). Heat shock protein 70 promotes cancer cell viability by safeguarding lysosomal integrity. Cell Cycle, 3(12), 1484-1485. doi:10.4161/cc.3.12.1287

15. Herrera, C., Sanchez, J., Torres, A., Bellido, C., Rueda, A., \& Alvarez, M. A. (2001). Early-acting cytokinedriven ex vivo expansion of mobilized peripheral blood CD34+ cells generates post-mitotic offspring with preserved engraftment ability in non-obese diabetic/severe combined immunodeficient mice. $\mathrm{Br}$ J Haematol, 114(4), 920-930. doi:10.1046/j.1365-2141.2001.02974.x

16. Hosseini, E., Ghasemzadeh, M., Kamalizad, M., \& Schwarer, A. P. (2017). Ex vivo expansion of CD3(depleted) cord blood-MNCs in the presence of bone marrow stromal cells; an appropriate strategy to provide functional NK cells applicable for cellular therapy. Stem Cell Res, 19, 148-155. doi:10.1016/j.scr.2017.01.010

17. Huenecke, S., Zimmermann, S. Y., Kloess, S., Esser, R., Brinkmann, A., Tramsen, L., . . Koehl, U. (2010). IL-2-driven regulation of NK cell receptors with regard to the distribution of CD16+ and CD16subpopulations and in vivo influence after haploidentical NK cell infusion. $J$ Immunother, 33(2), 200210. doi:10.1097/CJI.0b013e3181bb46f7

18. Jinnin, M., Ihn, H., \& Tamaki, K. (2006). Characterization of SIS3, a novel specific inhibitor of Smad3, and its effect on transforming growth factor-beta1-induced extracellular matrix expression. Mol Pharmacol, 69(2), 597-607. doi:10.1124/mol.105.017483

19. Judge, S. J., Murphy, W. J., \& Canter, R. J. (2020). Characterizing the Dysfunctional NK Cell: Assessing the Clinical Relevance of Exhaustion, Anergy, and Senescence. Front Cell Infect Microbiol, 10, 49. doi:10.3389/fcimb. 2020.00049

20. Kao, I. T., Yao, C. L., Kong, Z. L., Wu, M. L., Chuang, T. L., \& Hwang, S. M. (2007). Generation of natural killer cells from serum-free, expanded human umbilical cord blood CD34+ cells. Stem Cells Dev, 16(6), 1043-1051. doi:10.1089/scd.2007.0033

21. Klingemann, H. (2015). Challenges of cancer therapy with natural killer cells. Cytotherapy, 17(3), 245249. doi:10.1016/j.jcyt.2014.09.007

22. Lim, O., Jung, M. Y., Hwang, Y. K., \& Shin, E. C. (2015). Present and Future of Allogeneic Natural Killer Cell Therapy. Front Immunol, 6, 286. doi:10.3389/fimmu.2015.00286 
23. Mehta, R. S., Shpall, E. J., \& Rezvani, K. (2015). Cord Blood as a Source of Natural Killer Cells. Front Med (Lausanne), 2, 93. doi:10.3389/fmed.2015.00093

24. Michaud, A., Dardari, R., Charrier, E., Cordeiro, P., Herblot, S., \& Duval, M. (2010). IL-7 enhances survival of human CD56bright NK cells. J Immunother, 33(4), 382-390. doi:10.1097/CJI.0b013e3181cd872d

25. Miller, J. S., Tessmer-Tuck, J., Blake, N., Lund, J., Scott, A., Blazar, B. R., \& Orchard, P. J. (1997). Endogenous IL-2 production by natural killer cells maintains cytotoxic and proliferative capacity following retroviral-mediated gene transfer. Exp Hematol, 25(11), 1140-1148.

26. Mourcin, F., Grenier, N., Mayol, J. F., Lataillade, J. J., Sotto, J. J., Herodin, F., \& Drouet, M. (2005). Mesenchymal stem cells support expansion of in vitro irradiated CD34(+) cells in the presence of SCF, FLT3 ligand, TPO and IL3: potential application to autologous cell therapy in accidentally irradiated victims. Radiat Res, 164(1), 1-9. doi:10.1667/rr3384

27. Mu, Y. X., Zhao, Y. X., Li, B. Y., Bao, H. J., Jiang, H., Qi, X. L., .. Wu, X. Y. (2019). A simple method for in vitro preparation of natural killer cells from cord blood. BMC Biotechnol, 19(1), 80. doi:10.1186/s12896-019-0564-0

28. Multhoff, G. (2009). Activation of natural killer cells by heat shock protein 70. 2002. Int J Hyperthermia, 25(3), 169-175. doi:10.1080/02656730902902001

29. Ni, F., Sun, R., Fu, B., Wang, F., Guo, C., Tian, Z., \& Wei, H. (2013). IGF-1 promotes the development and cytotoxic activity of human NK cells. Nat Commun, 4, 1479. doi:10.1038/ncomms2484

30. Pinho, M. J., Punzel, M., Sousa, M., \& Barros, A. (2011). Ex vivo differentiation of natural killer cells from human umbilical cord blood CD34+ progenitor cells. Cell Commun Adhes, 18(3), 45-55. doi:10.3109/15419061.2011.610911

31. Qiao, Y., Liu, B., \& Li, Z. (2008). Activation of NK cells by extracellular heat shock protein 70 through induction of NKG2D ligands on dendritic cells. Cancer Immun, 8, 12.

32. Seo, H., Jeon, I., Kim, B. S., Park, M., Bae, E. A., Song, B., .. Kang, C. Y. (2017). IL-21-mediated reversal of NK cell exhaustion facilitates anti-tumour immunity in MHC class I-deficient tumours. Nat Commun, 8, 15776. doi:10.1038/ncomms 15776

33. Sharifzad, F., Mardpour, S., Mardpour, S., Fakharian, E., Taghikhani, A., Sharifzad, A., . . Ebrahimi, M. (2020). HSP70/IL-2 Treated NK Cells Effectively Cross the Blood Brain Barrier and Target Tumor Cells in a Rat Model of Induced Glioblastoma Multiforme (GBM). Int J Mol Sci, 21(7). doi:10.3390/ijms21072263

34. Skak, K., Frederiksen, K. S., \& Lundsgaard, D. (2008). Interleukin-21 activates human natural killer cells and modulates their surface receptor expression. Immunology, 123(4), 575-583. doi:10.1111/j.1365-2567.2007.02730.x

35. Souza-Fonseca-Guimaraes, F., Cursons, J., \& Huntington, N. D. (2019). The Emergence of Natural Killer Cells as a Major Target in Cancer Immunotherapy. Trends Immunol, 4O(2), 142-158. doi:10.1016/j.it.2018.12.003

36. Spanholtz, J., Preijers, F., Tordoir, M., Trilsbeek, C., Paardekooper, J., de Witte, T., ... Dolstra, H. (2011). Clinical-grade generation of active NK cells from cord blood hematopoietic progenitor cells for 
immunotherapy using a closed-system culture process. PLoS One, 6(6), e20740. doi:10.1371/journal.pone.0020740

37. Spanholtz, J., Tordoir, M., Eissens, D., Preijers, F., van der Meer, A., Joosten, I., . . Dolstra, H. (2010). High log-scale expansion of functional human natural killer cells from umbilical cord blood CD34positive cells for adoptive cancer immunotherapy. PLoS One, 5(2), e9221. doi:10.1371/journal.pone.0009221

38. Specht, H. M., Ahrens, N., Blankenstein, C., Duell, T., Fietkau, R., Gaipl, U. S., . . Multhoff, G. (2015). Heat Shock Protein 70 (Hsp70) Peptide Activated Natural Killer (NK) Cells for the Treatment of Patients with Non-Small Cell Lung Cancer (NSCLC) after Radiochemotherapy (RCTx) - From Preclinical Studies to a Clinical Phase II Trial. Front Immunol, 6, 162. doi:10.3389/fimmu.2015.00162

39. Tang, P. M., Zhou, S., Meng, X. M., Wang, Q. M., Li, C. J., Lian, G. Y., .. Lan, H. Y. (2017). Smad3 promotes cancer progression by inhibiting E4BP4-mediated NK cell development. Nat Commun, 8, 14677. doi:10.1038/ncomms14677

40. Vely, F., \& Vivier, E. (2005). Natural killer cell receptor signaling pathway. Sci STKE, 2005(292), cm6. doi:10.1126/stke. $2922005 \mathrm{~cm} 6$

41. Vivier, E., Nunes, J. A., \& Vely, F. (2004). Natural killer cell signaling pathways. Science, 306(5701), 1517-1519. doi:10.1126/science. 1103478

42. Wang, Q. M., Tang, P. M., Lian, G. Y., Li, C., Li, J., Huang, X. R., . . Lan, H. Y. (2018). Enhanced Cancer Immunotherapy with Smad3-Silenced NK-92 Cells. Cancer Immunol Res, 6(8), 965-977. doi:10.1158/2326-6066.CIR-17-0491

43. Watzl, C., \& Long, E. O. (2010). Signal transduction during activation and inhibition of natural killer cells. Curr Protoc Immunol, Chapter 11, Unit 11 19B. doi:10.1002/0471142735.im1109bs90

44. Wu, Y., Tian, Z., \& Wei, H. (2017). Developmental and Functional Control of Natural Killer Cells by Cytokines. Front Immunol, 8, 930. doi:10.3389/fimmu.2017.00930

45. Zhang, C., Wang, X. M., Li, S. R., Twelkmeyer, T., Wang, W. H., Zhang, S. Y., . . Tang, H. (2019). NKG2A is a NK cell exhaustion checkpoint for HCV persistence. Nat Commun, 10(1), 1507. doi:10.1038/s41467-019-09212-y

46. Zhang, Y., Shen, B., Guan, X., Qin, M., Ren, Z., Ma, Y., . . Jiang, Y. (2019). Safety and efficacy of ex vivo expanded CD34(+) stem cells in murine and primate models. Stem Cell Res Ther, 10(1), 173. doi:10.1186/s13287-019-1275-0

47. Zorzi, E., \& Bonvini, P. (2011). Inducible hsp70 in the regulation of cancer cell survival: analysis of chaperone induction, expression and activity. Cancers (Basel), 3(4), 3921-3956.

doi:10.3390/cancers3043921

\section{Table}

Due to technical limitations, table 1 is only available as a download in the Supplemental Files section. 


\section{Figures}

$\AA$

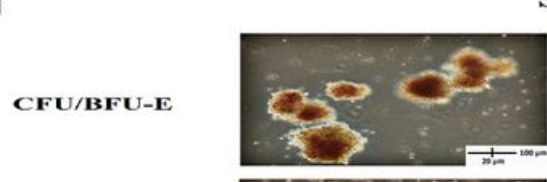

Ст-семм

Cru-gm
SFT
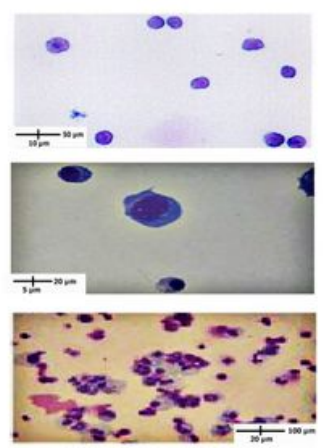
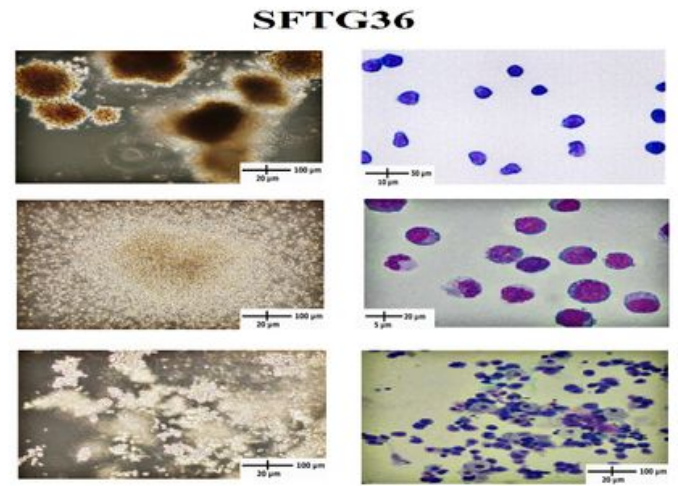

B

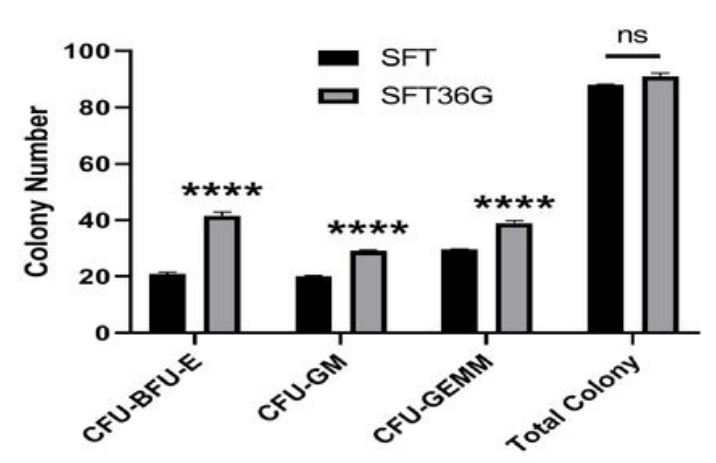

C

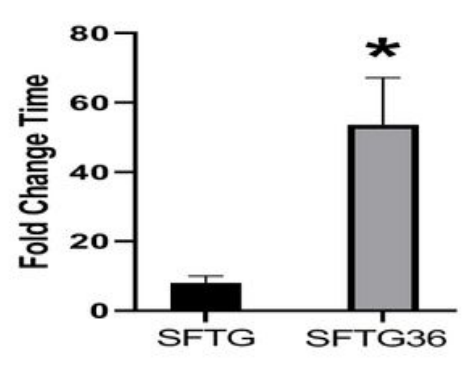

D

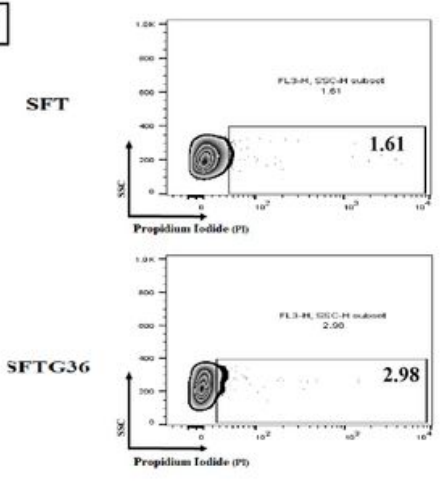

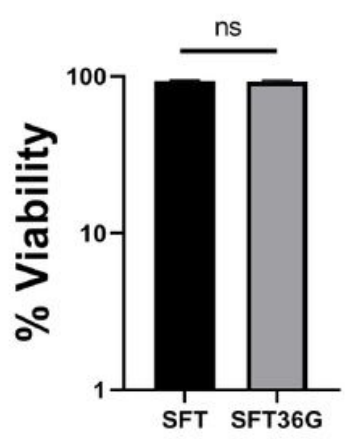
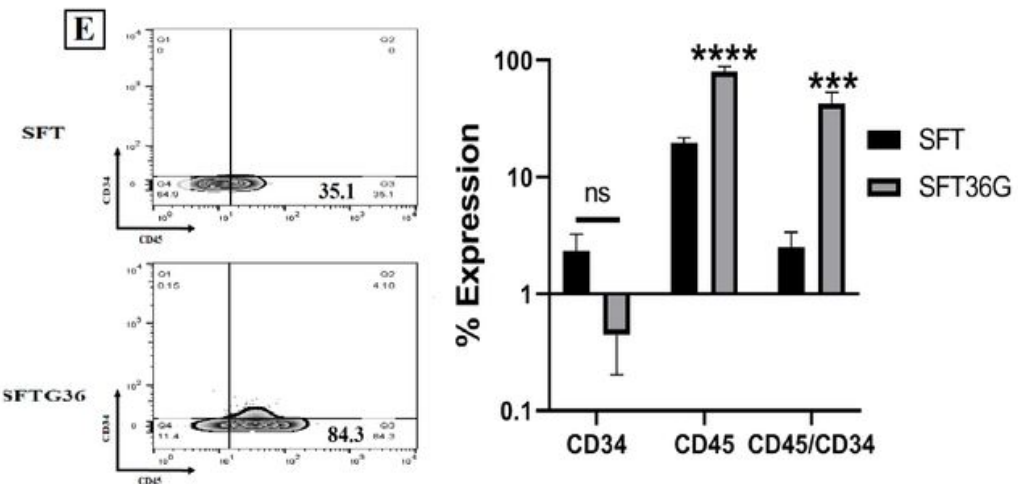

Figure 1

Characterization of the expanded HSCs CD34+ progenitor. A and B, The comparison of CFU assay results between the two groups treated with SFT and SFTG36 cocktail cytokines and morphological analysis by Wright-Giemsa staining. C-E, Both expanded cell populations were analyzed using flowcytometry and cell counting on the day 7. C, Analyses of fold expansion and D, cell viability of both groups. E, The phenotypic analysis of the expanded cells for CD45, CD34 and CD45/CD34 markers are shown as

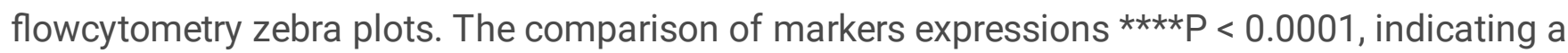
statistically significant increase in the expanded SFTG36 cell population compared to SFT cell population. $p<0.01$ was considered significant. Data are demonstrated as mean $\pm S E M, n=4$. 
A
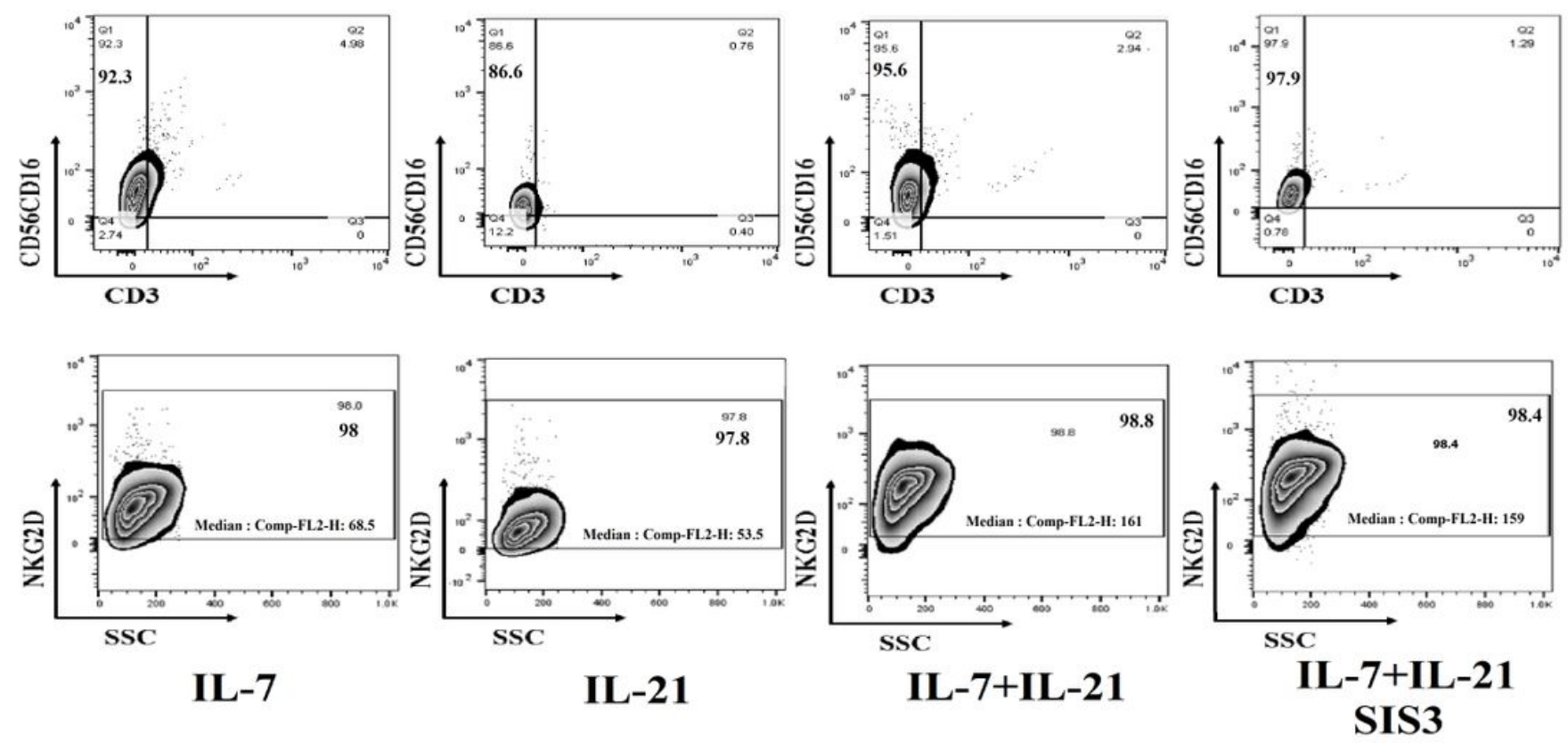

B

C
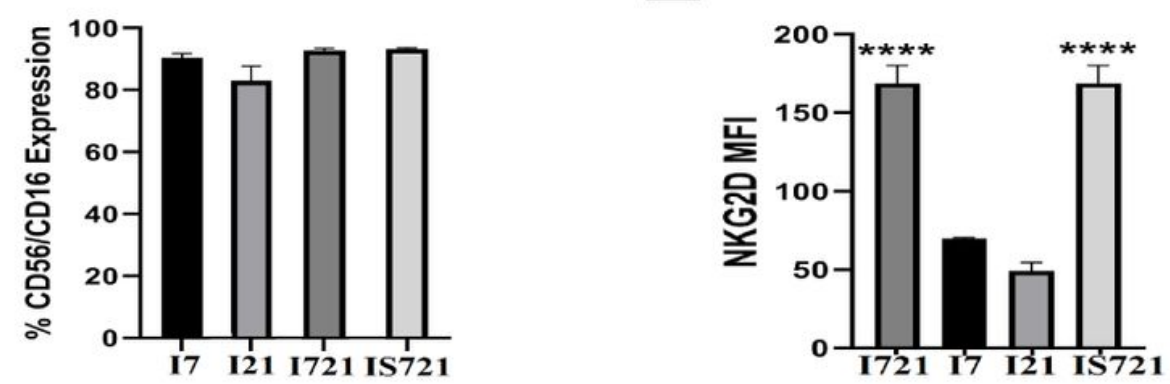

D

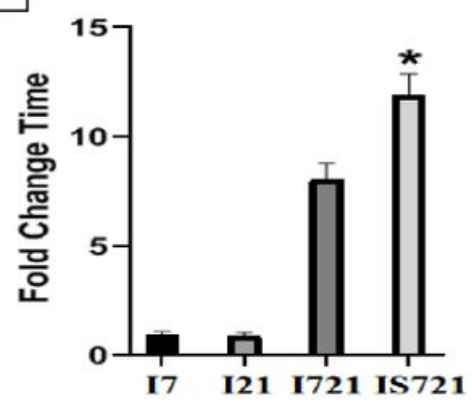

Figure 2

Comparison of characteristics of differentiated NK cells. A and B assessment of CD56/CD16 and CD3 markers expression among all 4 groups showed a significant expression difference in the groups IL-7+IL$21+$ IGF-1 $(P<0.01)$ and IL-7+IL-21+ IGF-1+SIS3 $(P<0.01)$. C, these 2 groups also demonstrated a significant difference in the expression of NKG2D compared to the other groups. $D$, the expansion potential analysis showed that IL-7+IL-21+ IGF-1+SIS3 group had the highest level of Fold expansion among all groups $(P<0.05)$. It should be noted that all expressions were assessed in the viable cells $(>90 \% \pm 3.00)$. Data are demonstrated as mean \pm SEM, $n=3$. 
$\mathbf{A}$

- NK Cell + IL-15

口 NK Cell + IL-15 + Hsp70
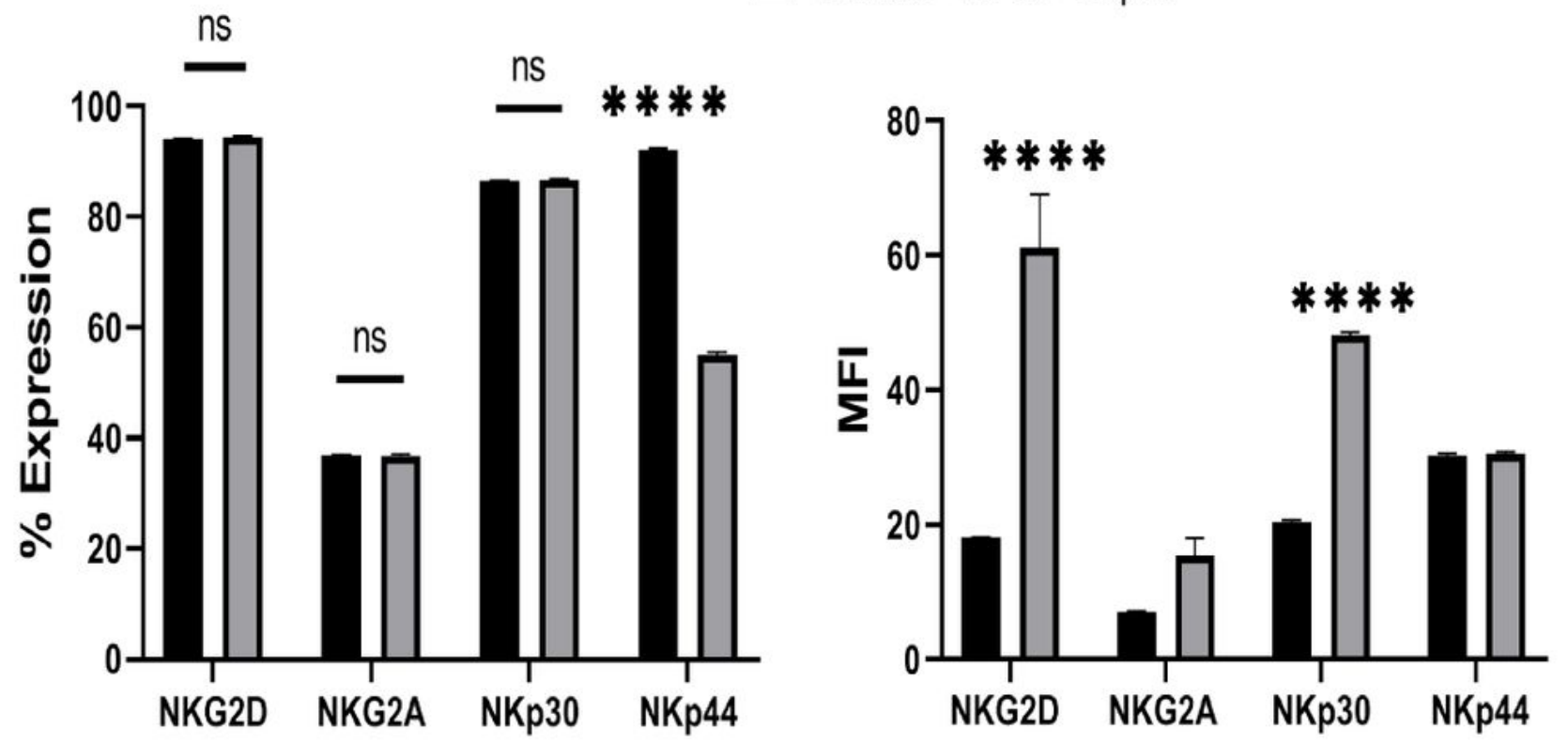

B

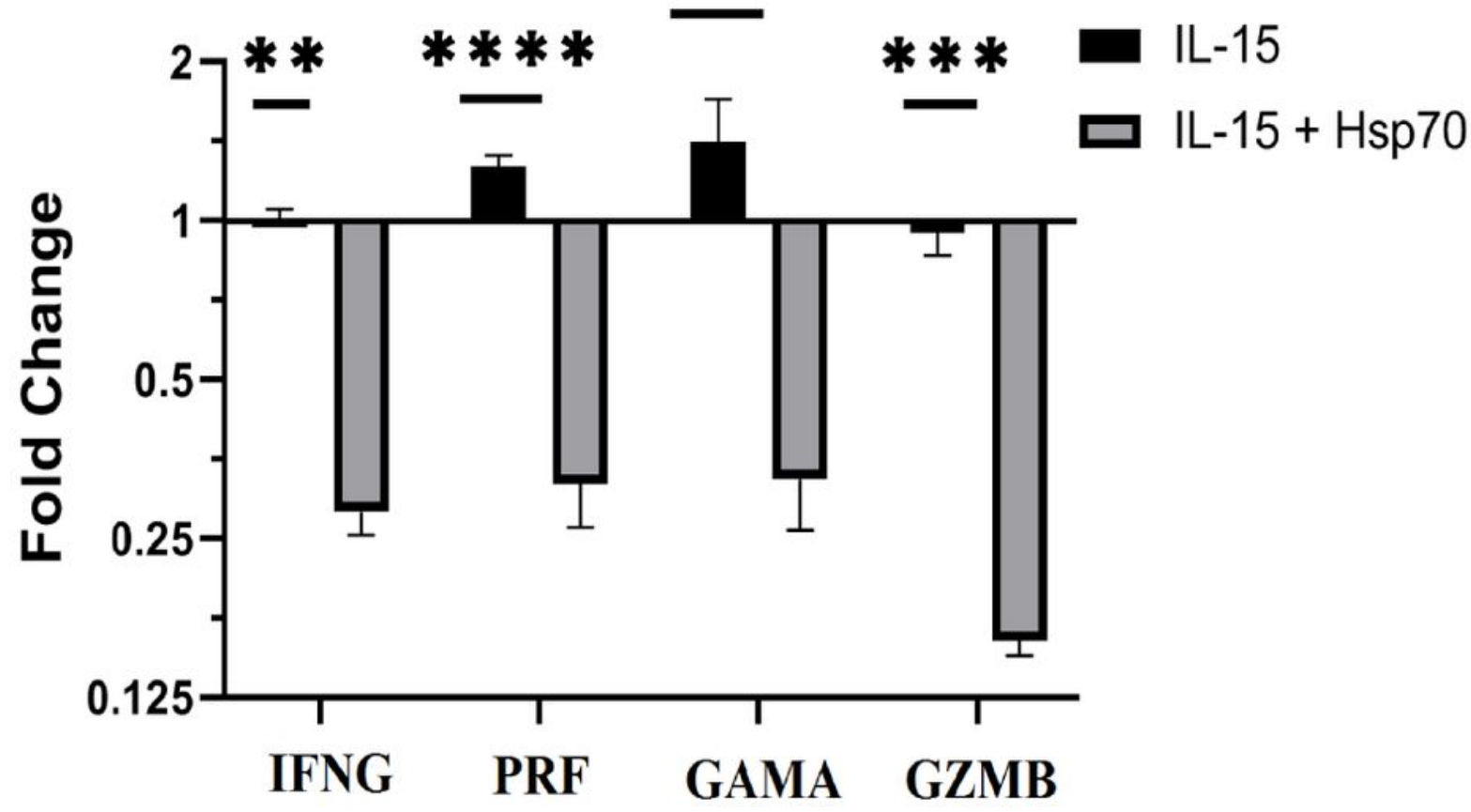

Figure 3

Comparison of characteristics of activated NK cells. A, Immunophenotyping of activated NK cells $(n=5)$. $B$, Analysis of the quantitative gene expression differences of Perforin, IFN- $\gamma$, Granzyme A and B between IL-15 and IL-15/Hsp70-activated NK cells on the 21st day $(n=3)$. Data are demonstrated as mean \pm SEM. 
NK Vs. K562
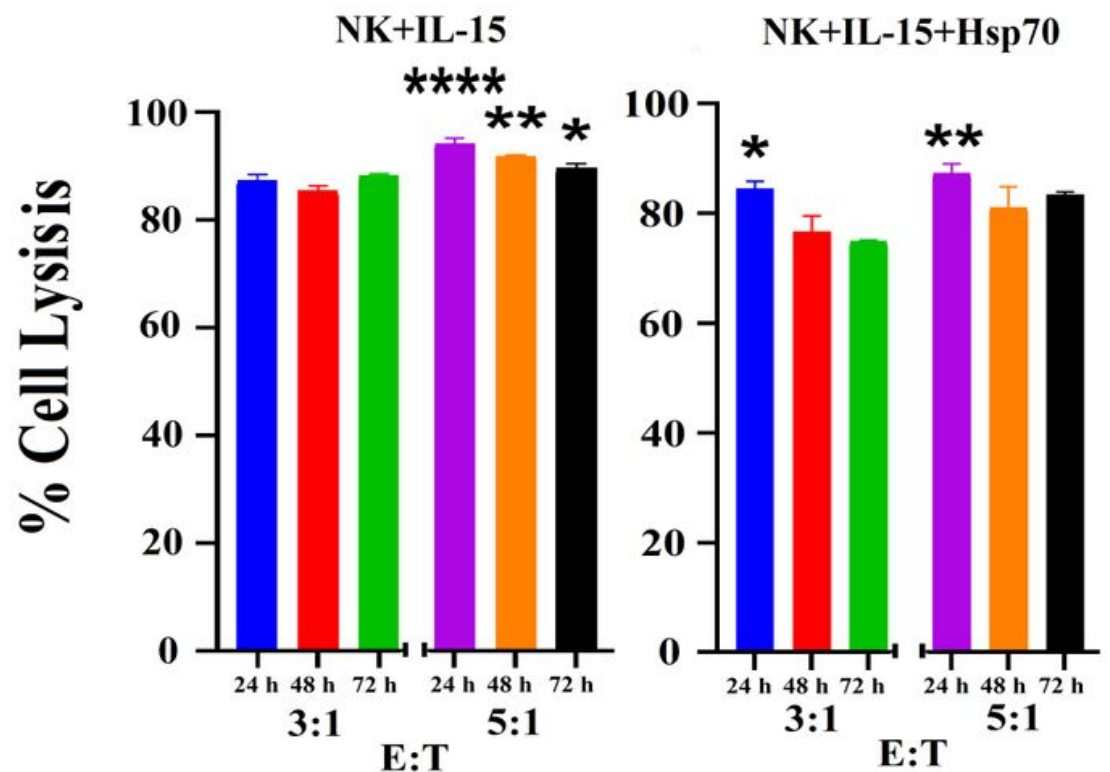

B

NK Vs. K562
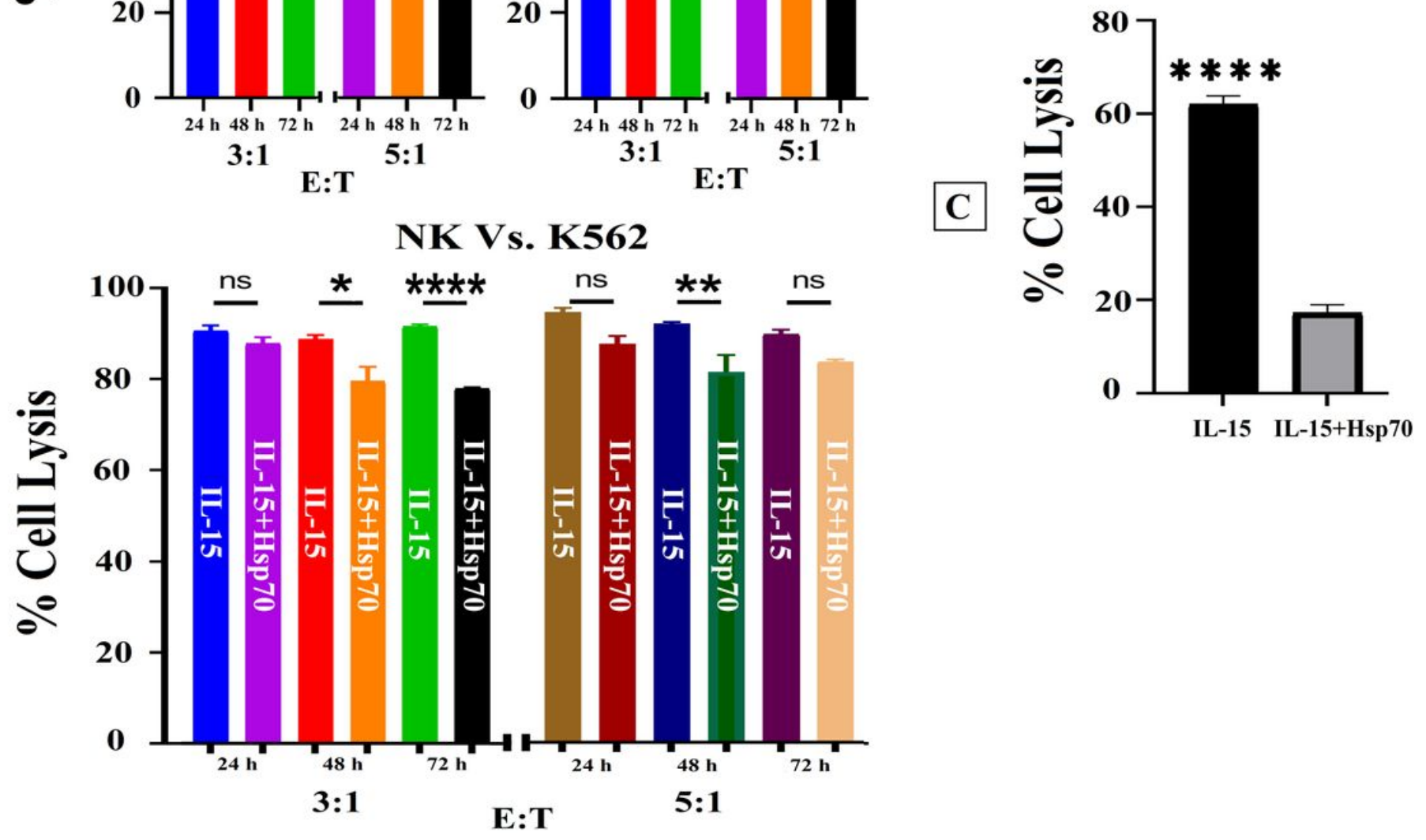

Figure 4

Cell lysis activities of NK cells. A, Comparison of the groups at different times and different ratios against K562 cells $(n=4)$. B, Specific cell lysis analysis of IL-15 and IL-15/Hsp70-activated NK cells against K562 cells at different times and effector ratios $(n=4)$. C, Cytotoxicity assay of IL-15 and IL-15/Hsp70activated NK cells at 3:1 ratio 48 hours after co-culture against SKOV3 cells $(n=7)$. The control cancer cells viability was about $90 \%$. Data are demonstrated as mean $\pm S E M, P>0.05$ which indicates no statistical significance. 

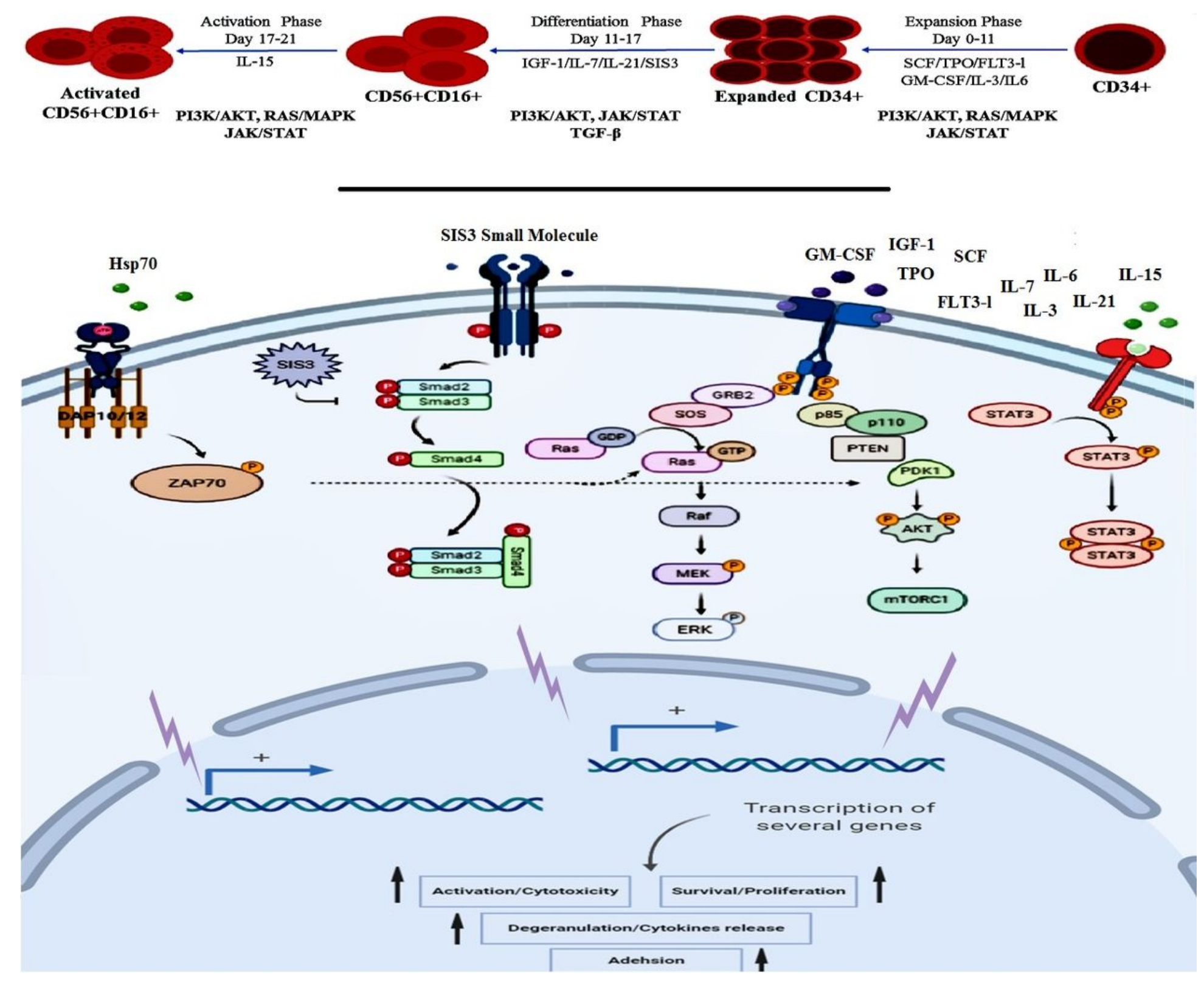

Figure 5

Conceptual schematic of the generation and activation of NK cells. In the present study, RAS/MAPK, PI3K/AKT, JAK/STAT pathways (involved in NK cells differentiation and activation) and TGF- $\beta$ pathway (involved in NK cells dysfunctionality) were induced and inhibited respectively.

\section{Supplementary Files}

This is a list of supplementary files associated with this preprint. Click to download.

- Supplementaryfigure1.Day0prePostMACS.jpeg

- Supplementaryfigure2.Diffımmunophenotyping.jpeg

- SupplementaryTable1.RealtimePCRprimersdescription.docx

- Table1.mediumexplantation.docx 